\title{
Higher-order QCD effects for associated WH production and decay at the LHC
}

\author{
Giancarlo Ferrera, $^{a}$ Massimiliano Grazzini ${ }^{b, 1}$ and Francesco Tramontano ${ }^{c}$ \\ ${ }^{a}$ Dipartimento di Fisica, Università di Milano and INFN — Sezione di Milano, \\ via Celoria 16, I-20133 Milan, Italy \\ ${ }^{b}$ Institut für Theoretische Physik, Universität Zürich, \\ Winterthurerstrasse 190, CH-8057 Zürich, Switzerland \\ ${ }^{c}$ Dipartimento di Fisica, Università di Napoli "Federico II" and INFN — Sezione di Napoli, \\ Complesso di Monte Sant'Angelo, via Cintia, I-80125 Naples, Italy \\ E-mail: giancarlo.ferrera@mi.infn.it, grazzini@physik.uzh.ch, \\ francesco.tramontano@na.infn.it
}

Abstract: We consider Standard Model Higgs boson production in association with a $W$ boson in hadron collisions. We supplement the fully exclusive perturbative computation of QCD radiative effects up to next-to-next-to-leading order (NNLO) with the computation of the decay of the Higgs boson into a $b \bar{b}$ pair at next-to-leading order (NLO). We consider the selection cuts that are typically applied in the LHC experimental analysis, and we compare our fixed-order predictions with the results obtained with the MC@NLO event generator. We find that NLO corrections to the $H \rightarrow b \bar{b}$ decay can be important to obtain a reliable $p_{T}$ spectrum of the Higgs boson, but that, in the cases of interest, their effect is well accounted for by the parton shower Monte Carlo. NNLO corrections to the production process typically decrease the cross section by an amount which depends on the detail of the applied cuts, but they have a mild effect on the shape of the Higgs $p_{T}$ spectrum. We also discuss the effect of QCD radiative corrections on the invariant mass distribution of the Higgs candidate.

KEYwords: QCD Phenomenology, Hadronic Colliders

ArXiv EPRINT: 1312.1669

\footnotetext{
${ }^{1}$ On leave of absence from INFN, Sezione di Firenze, Sesto Fiorentino, Florence, Italy.
} 


\section{Contents}

1 Introduction 1

2 Computation 3

3 Numerical results at $\sqrt{s}=8 \mathrm{TeV} \quad 4$

4 Numerical results at $\sqrt{s}=14 \mathrm{TeV} \quad 12$

$\begin{array}{lll}5 & \text { Summary } & 15\end{array}$

\section{Introduction}

The investigation of the origin of the electroweak symmetry breaking is one of the main goals of the physics program at the Large Hadron Collider (LHC). The detailed study of the scalar resonance recently discovered by the ATLAS and CMS experiments $[1,2]$ could lift the veil on the fundamental mechanism that gives mass to the known elementary particles.

One of the important production mechanisms of a light Higgs boson [3, 4] at hadron colliders is the associated production with a vector boson $V=W^{ \pm}, Z$ (also known as the Higgs-strahlung process). The vector boson provides a clean experimental signature, due to the presence of a high- $p_{T}$ lepton(s) and/or large missing transverse energy, and allows us to tag the $H \rightarrow b \bar{b}$ decay, which is characterized by a large branching fraction. This channel offers the opportunity to separately study the Higgs couplings to $W$ and $Z$ bosons. The $V H$ production was the main search channel for a light Higgs boson at the Tevatron, and lead to the observation of an excess of events [5] compatible with the scalar resonance observed at the LHC.

At the LHC the associated $V H$ production was considered less promising, due to the large backgrounds. This situation can be substantially improved by restricting the analysis to the so called boosted region, where the vector boson and/or the $b \bar{b}$ pair have a large transverse momentum, and possibly applying an extra light-jet veto [6]. This search strategy, however, significantly reduces the number of signal events, and its potential will be fully exploited only when the centre-of-mass energy $\sqrt{s}$ will reach 13 (14) TeV. At present, with the full LHC data set at $\sqrt{s}=7$ and $8 \mathrm{TeV}$ essentially analysed, ATLAS [7] sees no signal in this channel, with a signal strength, relative to that of the Standard Model (SM) Higgs boson, which is $\mu=0.2 \pm 0.5$ (stat.) \pm 0.4 (syst.). CMS [8] sees a (small) excess of events above the expected background with a local significance of $2.1 \sigma$, consistent with the expectation from the production of the SM Higgs boson. The signal strength corresponding to this excess is $\mu=1.0 \pm 0.5$.

The actual experimental analyses are based on complicated selection cuts and it is thus important to count on an accurate modelling of QCD radiation. In order to obtain good 
control of the efficiency of the selection cuts, and to assess whether the Monte Carlo tools correctly describe the relevant distributions, (fully) differential computations including the available radiative corrections are necessary.

The status of theoretical predictions for $V H$ production goes as follows. The NNLO QCD corrections for the $V H$ inclusive cross section were computed in [9], where all the Drell-Yan-like [10] contributions (plus the gluon induced heavy-quark mediated corrections for the $Z H$ case) were included. The quark induced heavy-quark mediated corrections for the $V H$ inclusive cross section were computed in [11] and found to be at the 1-3\% level at the LHC. Soft-gluon effects to $V H$ production have been considered in ref. [12]. A fully differential computation of NNLO QCD corrections for $W H$ production was presented in ref. [13], while in ref. [14] the NLO QCD corrections for both $W H$ production and $H \rightarrow b \bar{b}$ decay were combined. The NLO electroweak corrections for $W H$ production have been computed [15] and implemented in the fully exclusive numerical code HAWK. The computation of the fully differential $H \rightarrow b \bar{b}$ decay rate in NNLO QCD has been reported in ref. [16]. The inclusive $H \rightarrow b \bar{b}$ decay rate is known up to $\mathcal{O}\left(\alpha_{\mathrm{S}}^{4}\right)$ [17].

As far as Monte Carlo implementations are concerned, NLO corrections to $\mathrm{VH}$ production have been matched to the parton shower within the MC@NLO [18] framework in ref. [19] and within the POWHEG [20] framework in ref. [21]. Recently, an NLO simulation matched to the parton shower for $V H+1$ jet has been presented in ref. [22], and merged by using the method of ref. [23], with the corresponding $V H+0$ jet simulation. At present, the ATLAS analysis [7] is based on a Monte Carlo signal sample generated with PYTHIA8 [24], whereas the CMS analysis [8] uses POWHEG interfaced with HERWIG $++[25] .{ }^{1}$

As it was shown in ref. [13], even if the effect of higher orders QCD radiative corrections can be relatively modest on the inclusive cross section [9], its impact on the accepted cross section and the relevant kinematical distributions can be quite significant, in particular when severe selection cuts are applied, as in the boosted $V H$ analysis. The calculation of ref. [13] considered QCD corrections only to the production process $p p \rightarrow W H$, by neglecting QCD radiative effect in the $H \rightarrow b \bar{b}$ decay. Fully inclusive QCD effects in the $H \rightarrow b \bar{b}$ decay were taken into account by normalizing the $H \rightarrow b \bar{b}$ branching fraction to the result of ref. [27]. This should be a good approximation if one considers observables that are sufficiently inclusive over the extra radiation from the $b \bar{b}$ pair. The study of ref. [14], however, casts some doubts on this approximation, by showing that QCD effects from the decay can be relatively important, especially with the selection cuts used by the LHC experiments at $\sqrt{s}=8 \mathrm{TeV}$.

In this paper we extend and update the analysis presented in ref. [13] in two respects. As a first step towards a complete NNLO calculation of QCD corrections for $p p \rightarrow W H \rightarrow$ $l \nu b \bar{b}$, we supplement the NNLO calculation of ref. [13] with QCD corrections to the $H \rightarrow b \bar{b}$ decay up to NLO. As mentioned above, one important point is to understand the extent to which the QCD radiative effects are captured by the Monte Carlo generators used in the analysis. We thus compare our fixed order results with those obtained with the MC@NLO

\footnotetext{
${ }^{1}$ We note that HERWIG ++ includes the possibility to account for NLO corrections in both $V H$ production and $H \rightarrow b \bar{b}$ decay [26], but the Monte Carlo sample used by CMS is generated with a LO $H \rightarrow b \bar{b}$ decay.
} 
event generator, which includes radiation from the $b \bar{b}$ pair through the parton shower. Our analysis is performed both at $\sqrt{s}=8$ and $14 \mathrm{TeV}$, by using the selection cuts typically applied by the ATLAS and CMS collaborations.

This paper is organized as follows. In section 2 we describe our calculation. In section 3 we present our results at the LHC with $\sqrt{s}=8 \mathrm{TeV}$, and in section 4 we consider the case of the LHC with $\sqrt{s}=14 \mathrm{TeV}$. In section 5 we summarize our results.

\section{Computation}

In this section we introduce the theoretical framework adopted in our calculation. We consider the inclusive hard scattering process

$$
p p \rightarrow W H+X \rightarrow W b \bar{b}+X
$$

where the Higgs boson $H$, which subsequently decays into a $b \bar{b}$ pair, is produced together with a $W$ boson. $^{2}$ Our goal is to construct the most precise predictions for the distributions that are sensitive to selection cuts and vetoes on the jet activity in both the production and decay stages of the Higgs boson.

The production differential cross section for the process (2.1) can be written as:

$$
d \sigma_{p p \rightarrow W H+X}=d \sigma_{p p \rightarrow W H+X}^{(0)}+d \sigma_{p p \rightarrow W H+X}^{(1)}+d \sigma_{p p \rightarrow W H+X}^{(2)}+\mathcal{O}\left(\alpha_{\mathrm{S}}^{3}\right),
$$

where $d \sigma^{(0)}$ is the LO contribution, and $d \sigma^{(1)}$ and $d \sigma^{(2)}$ the NLO and NNLO correction, respectively. Analogously, the $H \rightarrow b \bar{b}$ differential decay rate is

$$
d \Gamma_{H \rightarrow b \bar{b}}=d \Gamma_{H \rightarrow b \bar{b}}^{(0)}+d \Gamma_{H \rightarrow b \bar{b}}^{(1)}+d \Gamma_{H \rightarrow b \bar{b}}^{(2)}+\mathcal{O}\left(\alpha_{\mathrm{S}}^{3}\right) .
$$

By using the narrow width approximation, the differential cross section for (2.1) can be written as

$$
d \sigma_{p p \rightarrow W H+X \rightarrow W b \bar{b}+X}=\left[\sum_{k=0}^{\infty} d \sigma_{p p \rightarrow W H+X}^{(k)}\right] \times\left[\frac{\sum_{k=0}^{\infty} d \Gamma_{H \rightarrow b \bar{b}}^{(k)}}{\sum_{k=0}^{\infty} \Gamma_{H \rightarrow b \bar{b}}^{(k)}}\right] \times \operatorname{Br}(H \rightarrow b \bar{b}) .
$$

Through eq. (2.4) we can exploit the precise prediction of the Higgs boson branching ratio into $b$ quarks $\operatorname{Br}(H \rightarrow b \bar{b})$, reported in [27], by which we normalize the contributions to the differential decay rate of the Higgs boson. We can consider various approximations of eq. (2.4). We first consider NLO corrections to the production process and ignore QCD corrections to the decay, by defining

$$
d \sigma_{p p \rightarrow W H+X \rightarrow W b \bar{b}+X}^{\mathrm{NLO}(\mathrm{prod})+\mathrm{LO}(\mathrm{dec})}=\left[d \sigma_{p p \rightarrow W H+X}^{(0)}+d \sigma_{p p \rightarrow W H+X}^{(1)}\right] \times d \Gamma_{H \rightarrow b \bar{b}}^{(0)} / \Gamma_{H \rightarrow b \bar{b}}^{(0)} \times B r(H \rightarrow b \bar{b}) .
$$

\footnotetext{
${ }^{2}$ The leptonic decay of the $W$ boson (including spin correlations) does not lead to complications and is understood in this section to simplify the notation.
} 
By including NLO corrections to the $H \rightarrow b \bar{b}$ decay we define

$$
\begin{aligned}
& d \sigma_{p p \rightarrow W H+X \rightarrow W b \bar{b}+X}^{\mathrm{NLO}(\mathrm{prod})+\mathrm{NLO}(\mathrm{dec})} \\
& \quad=\left[d \sigma_{p p \rightarrow W H}^{(0)} \times \frac{d \Gamma_{H \rightarrow b \bar{b}}^{(0)}+d \Gamma_{H \rightarrow b \bar{b}}^{(1)}}{\Gamma_{H \rightarrow b \bar{b}}^{(0)}+\Gamma_{H \rightarrow b \bar{b}}^{(1)}}+d \sigma_{p p \rightarrow W H+X}^{(1)} \times \frac{d \Gamma_{H \rightarrow b \bar{b}}^{(0)}}{\Gamma_{H \rightarrow b \bar{b}}^{(0)}}\right] \times B r(H \rightarrow b \bar{b}),
\end{aligned}
$$

which represents the complete NLO calculation considered in ref. [14]. We point out here that at the first order in $\alpha_{\mathrm{S}}$ the factorization between production and decay is indeed exact because of colour conservation. In other words the interference of QCD radiation in Higgs boson production and decay stages vanishes at this order. This property does not hold beyond $\mathcal{O}\left(\alpha_{\mathrm{S}}\right)$.

As a first step towards a complete NNLO calculation we consider the following approximation of eq. (2.4)

$$
\begin{aligned}
d \sigma_{p p \rightarrow W H+X \rightarrow l \nu b \bar{b}+X}^{\mathrm{NNLO}(\mathrm{prod})+\mathrm{NLO}(\mathrm{dec})}=[ & d \sigma_{p p \rightarrow W H}^{(0)} \times \frac{d \Gamma_{H \rightarrow b \bar{b}}^{(0)}+d \Gamma_{H \rightarrow b \bar{b}}^{(1)}}{\Gamma_{H \rightarrow b \bar{b}}^{(0)}+\Gamma_{H \rightarrow b \bar{b}}^{(1)}} \\
& \left.+\left(d \sigma_{p p \rightarrow W H+X}^{(1)}+d \sigma_{p p \rightarrow W H+X}^{(2)}\right) \times \frac{d \Gamma_{H \rightarrow b \bar{b}}^{(0)}}{\Gamma_{H \rightarrow b \bar{b}}^{(0)}}\right] \times B r(H \rightarrow b \bar{b}) .
\end{aligned}
$$

In eq. (2.7) we include QCD corrections to the production stage up to NNLO, and the Higgs decay is treated up to NLO. Although this is not a fully consistent approximation, since it neglects some $\mathcal{O}\left(\alpha_{\mathrm{S}}^{2}\right)$ contributions in eq. (2.4), we believe it captures the relevant radiative effects (see discussion below).

The NNLO computation for the production process [13] is performed using the subtraction method proposed in [28]. This method allows us to compute up to NNLO contributions in QCD for the whole class of hadronic collisions producing a colourless final state at LO and it has been successfully applied to the computation of NNLO corrections to several hadronic processes [28-31].

The $H \rightarrow b \bar{b}$ decay at NLO is computed by using the dipole subtraction method [3234] and is included in a fully differential numerical code both for massless and massive $b$ quarks. We point out that in the on shell scheme the heavy-quark mass dependence leads to large logarithmic terms of the form $\ln m_{H} / m_{b}$, which render the whole $H \rightarrow b \bar{b}$ decay rate infrared unsafe. To correctly recover the $m_{b} \rightarrow 0$ limit these logarithmic terms must be absorbed into the running $H b \bar{b}$ Yukawa coupling [35, 36]. With this treatment the massless and massive computations produce in practice almost identical results, ${ }^{3}$ and in the next section we thus limit ourselves to consider the massless case.

\section{Numerical results at $\sqrt{s}=8 \mathrm{TeV}$}

In this section we present numerical results at the LHC, in the case $\sqrt{s}=8 \mathrm{TeV}$. We thus consider $W H$ production in $p p$ collisions followed by the $W \rightarrow l \nu_{l}$ and $H \rightarrow b \bar{b}$

\footnotetext{
${ }^{3}$ We note that the authors of ref. [14] do not absorb these large logarithmic terms into the $H b \bar{b}$ coupling and this leads to differences in the quantitative results of the two NLO computations.
} 
decays. We first focus on the $p_{T}$ spectrum of the Higgs candidate, whose knowledge is particularly important in the experimental analysis, and then we present our results for the corresponding invariant mass distribution. We use the so called $G_{\mu}$ scheme for the electroweak couplings, where the input parameters are $G_{F}, m_{Z}, m_{W}$. In particular we use the following values: $G_{F}=1.16637 \times 10^{-5} \mathrm{GeV}^{-2}, m_{Z}=91.1876 \mathrm{GeV}, m_{W}=80.399 \mathrm{GeV}$ and $\Gamma_{W}=2.085 \mathrm{GeV}$. The mass of the SM Higgs boson is set to $m_{H}=125 \mathrm{GeV}$ and the width to $\Gamma_{H}=4.070 \mathrm{MeV}[27]$. The $W H$ production cross section is computed up to NNLO by using the calculation of ref. [13], including the leptonic decay of the $W$ boson, in both the $W^{+} \rightarrow l^{+} \nu_{l}$ and $W^{-} \rightarrow l^{-} \bar{\nu}_{l}$ channels. We compute the $H \rightarrow b \bar{b}$ decay up to NLO QCD and we normalize the $H b \bar{b}$ Yukawa coupling such that the value of the branching ratio is $\operatorname{Br}(H \rightarrow b \bar{b})=0.578$ [27]. The fixed order results are compared with the results obtained with the MC@NLO 4.09 event generator [18], normalized to the same $H \rightarrow b \bar{b}$ branching ratio, and without underlying event.

As for the parton distribution functions (PDFs) we use the NNPDF2.3 PDF sets [37], with densities and $\alpha_{\mathrm{S}}$ evaluated at each corresponding order (i.e., we use $(n+1)$-loop $\alpha_{\mathrm{S}}$ at $\mathrm{N}^{n} \mathrm{LO}$, with $\left.n=0,1,2\right)$ and with $\alpha_{\mathrm{S}}\left(m_{Z}\right)=0.118$. In the fixed order calculations the central values of the renormalization and factorization scales are fixed to the value $\mu_{R}=\mu_{F}=m_{W}+m_{H}$ while the central value of the renormalization scale for the $H \rightarrow b \bar{b}$ coupling is set to the value $\mu_{r}=m_{H}$. In the $\mathrm{MC} @ \mathrm{NLO}$ simulation the central scale is the default scale, the transverse mass of the $W H$ system. Jets are reconstructed with the anti- $k_{T}$ algorithm with $R=0.4$ [38] and with a transverse momentum $p_{T}^{j}>20 \mathrm{GeV}$. In order to simulate the experimental analysis for the Higgs search in this channel, we require exactly two $(R)$ separated $b$-jets each with $p_{T}^{b}>30 \mathrm{GeV}$ and $\left|\eta_{b}\right|<2.5$. In the fixed-order calculation a jet is considered a $b$-jet if it contains at least one $b$-quark. In the MC@NLO simulation this is achieved by requiring that, after hadronization, the jet contains at least one $B$-hadron.

We start the presentation of our results by considering the inclusive $W H$ selection of the $b$-jet pair. Note that thanks to eq. (2.4) and to the normalization of the $H \rightarrow b \bar{b}$ coupling, the prediction for the total cross-section is insensitive to the higher-order corrections to the $H \rightarrow b \bar{b}$ decay for a completely inclusive quantity: this is a valuable check of the implementation of the NLO corrections to the Higgs boson decay (still we can observe differences in the shape of distributions). In this inclusive case the only effective selection cuts are the minimum value of the transverse momentum used in the jet definition and the cuts which define the separated $b$-jets. In figure 1 (left panel) we show the QCD predictions at NLO (with and without NLO corrections to the $H \rightarrow b \bar{b}$ decay, see eqs. (2.5), (2.6)), at NNLO (see eq. (2.7)) and from MC@NLO, for the transverse-momentum distribution of the $b$-jets pair $p_{T}^{b \bar{b}}=\left|\vec{p}_{T}^{b}+\vec{p}_{T}^{\bar{b}}\right|$. In the $p_{T}^{b \bar{b}}=0$ bin we collect the events which do not fulfil the selection cuts. Here and in the following we take the complete NLO result (see eq. (2.6)) as reference theoretical prediction and in figure 1 (right panel) we plot the NLO, NNLO and MC@NLO $p_{T}$ distributions normalized to the NLO result, with their scale uncertainty band, which is obtained as follows. In the fixed order calculations we vary $\mu_{F}=\mu_{R}$ between $\left(m_{H}+m_{W}\right) / 2$ and $2\left(m_{H}+m_{W}\right)$ and, simultaneously, we vary the decay scale $\mu_{r}$ between $m_{H} / 2$ and $2 m_{H}$. In MC@NLO $\mu_{F}=\mu_{R}$ is varied by a factor of two around the central scale, the transverse mass of the $W H$ system. 

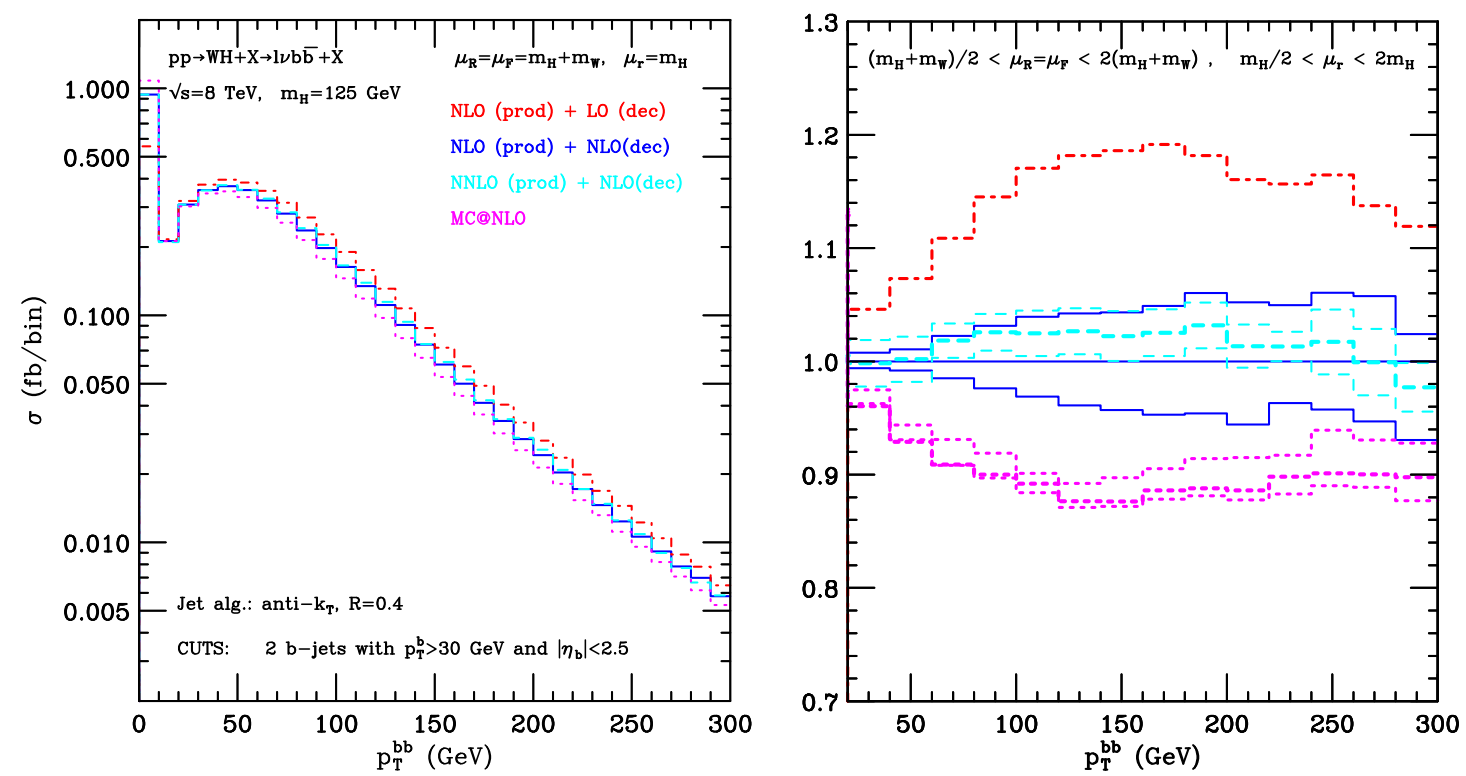

Figure 1. Left panel: transverse-momentum distribution of the $b$-jets pair computed at NLO with LO decay (red dot-dashes), NLO with NLO decay (blue solid), NNLO with NLO decay (cyan dashes) and with MC@NLO (magenta dots). Right panel: the same distributions normalized to the full NLO result. The NLO, NNLO and MC@NLO uncertainty bands are also shown. No cuts except the $b$-jet selection are applied.

By comparing the different spectra in figure 1 we see that the hardest is the NLO one (with LO $H \rightarrow b \bar{b}$ decay), with a selection efficiency of the $b$-jet pair of $88 \%$. If we consider the full NLO corrections, the spectrum becomes softer and the efficiency decreases to $79 \%$. This is not unexpected since, generally speaking, hard real emissions from the $b \bar{b}$ pair reduce the $p_{T}^{b \bar{b}}$ of the event and increase the probability that the $b$-quark radiating a hard gluon could fail the $p_{T}^{b}>30 \mathrm{GeV}$ threshold. This situation does not change significantly if we further consider the NNLO corrections for the production: we observe only a slight increase of the accepted cross section, at the $1 \%$ level. The effect of scale variations at NLO (NNLO) is of the order of about $\pm 2 \%( \pm 1 \%)$ on the accepted cross section, but it increases at high $p_{T}$, where it can be of $\mathcal{O}( \pm 5 \%)(\mathcal{O}( \pm 3 \%))$. The MC@NLO prediction, besides the NLO plus parton shower effects for the production, includes radiation from the $b \bar{b}$ pair due to the parton shower. In this case, we observe that even if the matrix elements for the $H \rightarrow b \bar{b}$ decay have a LO accuracy, the effect of the shower is qualitatively similar to (but quantitative larger than) the NLO corrections to the decay: the spectrum is softer and the efficiency reaches the $75 \%$ level. The physical picture is the one discussed before: parton emissions from the $b \bar{b}$ pair reduce the $p_{T}^{b \bar{b}}$ of the event and decrease the efficiency.

We now proceed to consider a more realistic situation in which we apply selection cuts similar to those used by ATLAS and CMS in their analysis. At $\sqrt{s}=8 \mathrm{TeV}$ and with the integrated luminosity accumulated, it is not really possible to perform a boosted analysis like that proposed in ref. [6]. The strategy of the Higgs boson search in this channel is thus to apply less stringent selection cuts, which aim at having the Higgs and the $W$ boson at relatively large $p_{T}$, and almost back to back, to reduce the $t \bar{t}$ background. 

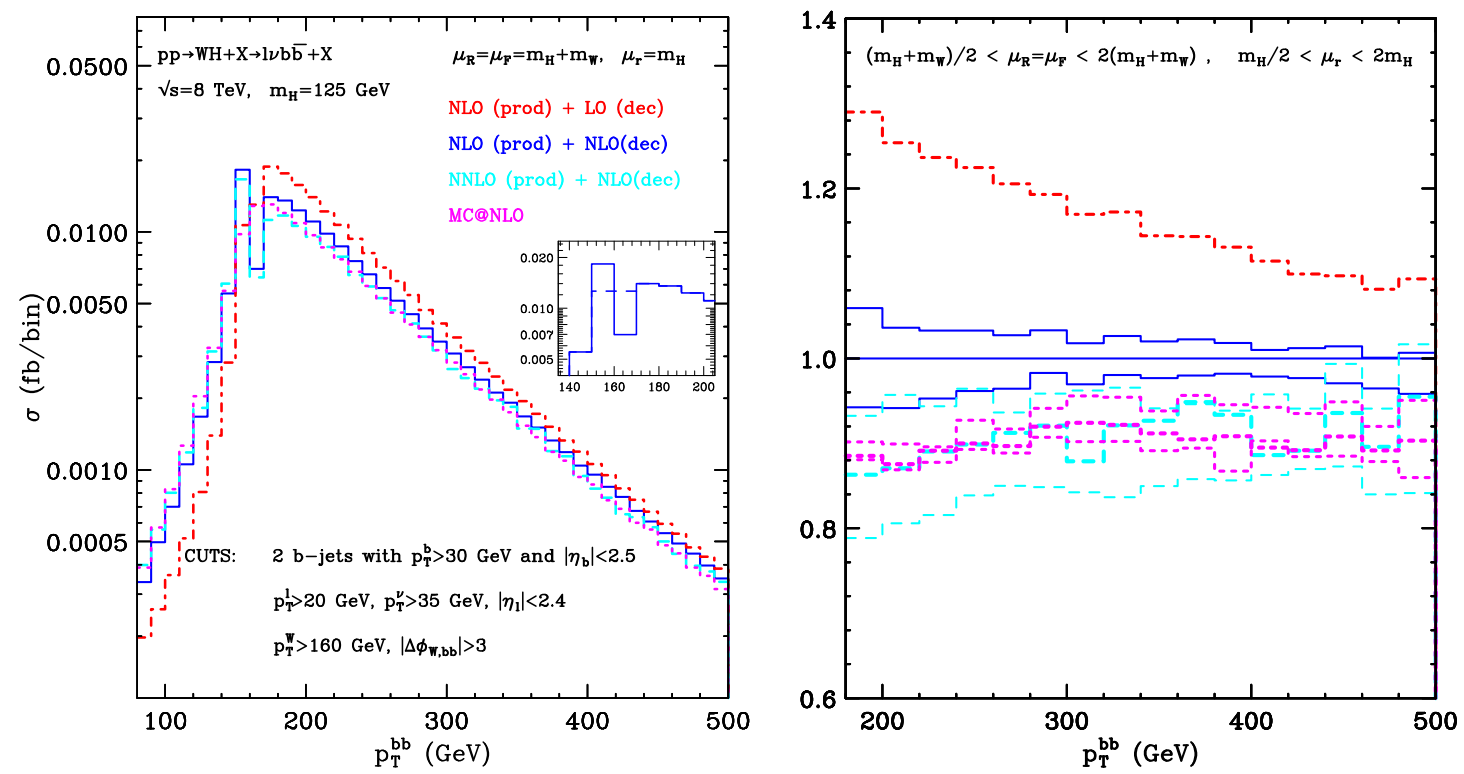

Figure 2. As in figure 1 but when selection cuts are applied. The inset plot shows the region around $p_{T}^{b b} \sim 160 \mathrm{GeV}$.

In particular, we consider here the following cuts. The charged lepton is required to have transverse momentum $p_{T}^{l}>20 \mathrm{GeV}$ and pseudorapidity $\left|\eta_{l}\right|<2.4$; the missing transverse momentum of the event is required to be $p_{T}^{\nu}>35 \mathrm{GeV}$. The $W$ boson must have a transverse momentum $p_{T}^{W}>160 \mathrm{GeV}$ and is required to be almost back-to-back with the Higgs candidate. To achieve this condition the azimuthal separation of the $W$ boson with the $b \bar{b}$ pair must fulfil $\left|\Delta \phi_{W, b b}\right|>3$. The selection on $p_{T}^{W}$ is important to improve the signal-to-background ratio: an analogous cut on the Higgs boson can be imposed by focusing on the large $p_{T}$ region in the $p_{T}^{b \bar{b}}$ distribution.

In figure 2 we study the $p_{T}^{b \bar{b}}$ distribution of the Higgs candidate. As above we consider QCD predictions at NLO (with and without corrections to the $H \rightarrow b \bar{b}$ decay), at NNLO (with NLO decay) and from MC@NLO. The corresponding cross sections and scale uncertainties are reported in the first row of table 1 . As in figure 1, in the right panel of figure 2 we plot the $p_{T}$ spectra normalized to the full NLO result.

As in the inclusive case the hardest spectrum is the NLO one (with LO $H \rightarrow b \bar{b}$ decay), with an accepted cross section which is only $4 \%$ with respect to the inclusive one (the bulk of the reduction is due to the tight cut on $p_{T}^{W}$ ). When including the NLO corrections to the $H \rightarrow b \bar{b}$ decay the spectrum becomes softer and the accepted cross section is further reduced by $12 \%$.

We observe from figure 2 that the inclusion of the NLO corrections produces instabilities around the region where $p_{T}^{b \bar{b}}=160 \mathrm{GeV}$. The origin of such instabilities is of Sudakov type [39]: at LO the $p_{T}^{W}>160 \mathrm{GeV}$ constraint imposes a kinematical boundary on the $p_{T}^{b \bar{b}}$ spectrum, and perturbative contribution at higher orders produce integrable logarithmic singularities around such boundary. The way to solve these perturbative instabilities is to perform an all-order resummation of the soft-gluon contributions which renders the distri- 
bution smooth in the vicinity of the boundary. The effects of soft-gluon resummation can be mimicked by considering a more inclusive observable i.e. by increasing the bin size of the distribution around the critical point. The effect of the smearing obtained in this way can be seen in the inset plot of figure 2 (dashed line).

We also observe that the NLO corrections to the decay below the $p_{T}^{b \bar{b}}=160 \mathrm{GeV}$ boundary are particularly large (reaching, for $p_{T}^{b \bar{b}} \sim 120$, the $100 \%$ level with respect to the cross section with NLO corrections for the production only). This is not unexpected, since in this region of transverse momenta, the $\mathcal{O}\left(\alpha_{\mathrm{S}}\right)$ correction to the Higgs boson decay contributes as a leading order term. Contrary to the inclusive case the NNLO corrections for the production are not negligible: the spectrum becomes softer and the accepted cross section is further reduced by $9 \%$.

Comparing the fixed order predictions to the MC@NLO result we observe that the effect of the shower is quantitative very similar to the effect of the NNLO corrections for the production plus NLO for the Higgs decay (with the exclusion of the region around the LO kinematical boundary discussed before). Moreover we note that the MC@NLO prediction around the LO kinematical boundary has a smooth behaviour, without the instabilities of the fixed order case. This is because the effective resummation of the Sudakov logarithms implemented in the shower algorithm permits a more reliable description of the region around the boundary.

The NLO scale uncertainties are $\mathcal{O}( \pm 10 \%)$ in the region $p_{T} \lesssim 200 \mathrm{GeV}$ and then decrease to the $\mathcal{O}( \pm 5 \%)$ level or smaller for higher values of $p_{T}$. From figure 2 (right panel) we conclude that the inclusion of NLO corrections to the Higgs decay is important to obtain a reliable shape of the $p_{T}$ spectrum. The MC@NLO prediction, on the other hand, even without the NLO corrections to the decay, describes the shape of the spectrum rather well. We also conclude that the NLO scale uncertainty is in this case too small to be considered as a true uncertainty from missing higher order contributions, since both the NNLO and MC@NLO results lie outside the NLO band. The NNLO uncertainty band is in turn larger than the NLO one, being at the $\pm 7-8 \%$ level, and marginally overlaps with the latter. The NNLO and MC@NLO results are perfectly compatible within the uncertainties.

To improve the background rejection, a veto on extra jet radiation is typically used in the analyses. In figure 3 we consider the case in which, besides the cuts considered above, events with additional jets with $p_{T}^{j}>20 \mathrm{GeV}$ and pseudorapidity $\left|\eta_{j}\right|<2.4$ are rejected. The corresponding cross sections and scale uncertainties are reported in the second row of table 1 . In order of increasing sensitivity, the effect of the jet veto is to reduce the accepted cross section by $25 \%$ at NLO (production only), by $33 \%$ for MC@NLO, by $41 \%$ at full NLO accuracy and by $44 \%$ at the NNLO. The reason of such sensitivity is the different content of radiative corrections which are present in the calculations. Most sensitive to the jet veto is the NNLO distribution (with NLO Higgs decay) where up to two hard emissions from the initial state and one hard emission from the final states are considered. As a result the jet veto produces a different behaviour of the distributions with respect of the situation in figure 2. In particular we observe that the full NLO result is very close to the MC@NLO prediction while the inclusion of the NNLO corrections for the production further reduces the accepted cross section by $10 \%$ (see table 1 ). 

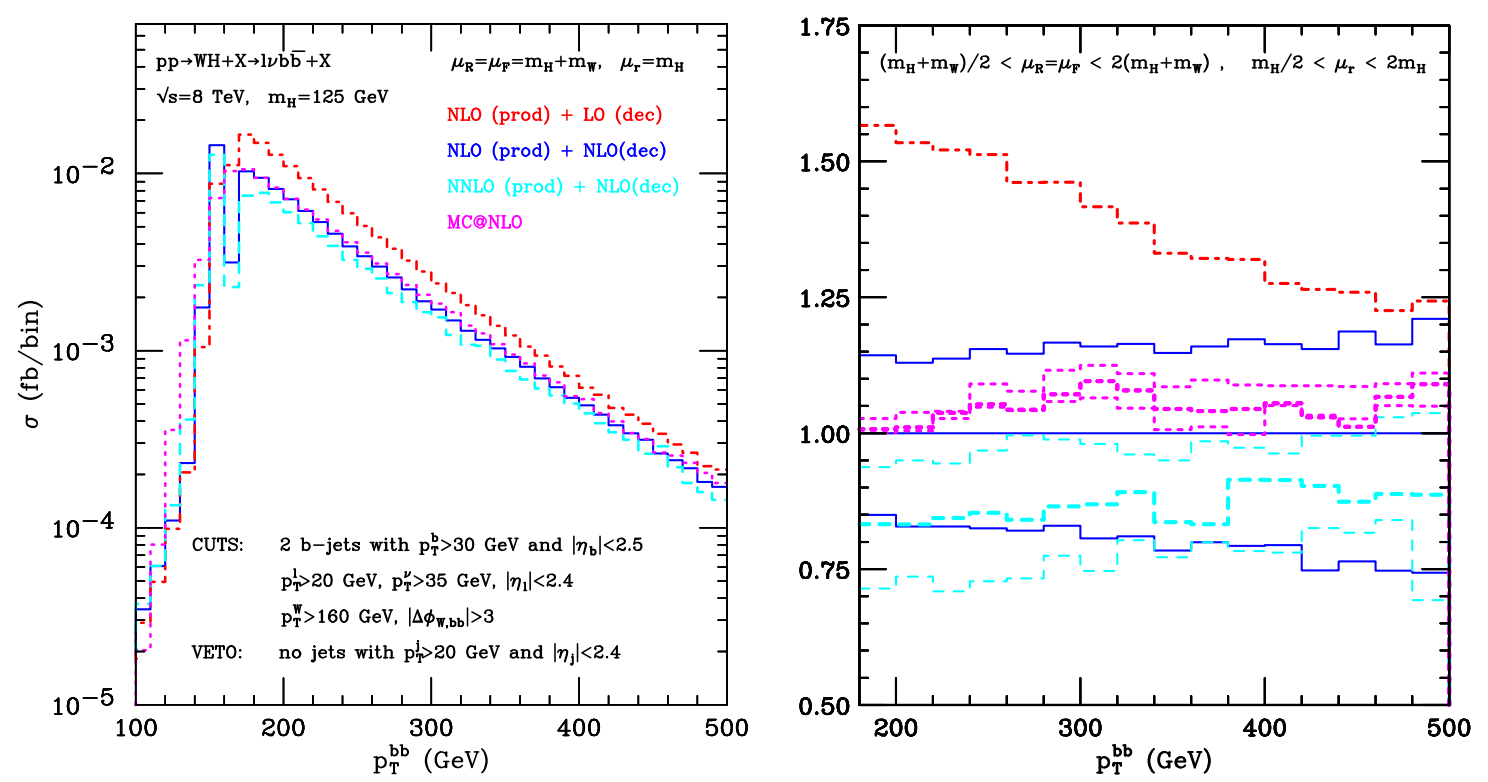

Figure 3. As in figure 2 but with an additional veto on light jets.

We add few comments on the stability of the perturbative results when a jet veto is applied [40]. As is well known, when a generic system of high-mass $M$ is produced in hadronic collisions, a veto on jets with $p_{T}>p_{T}^{\text {veto }}$ leads to potential instabilities in the perturbative expansion, since the cancellation between real and virtual contributions is unbalanced. The typical scale of the accompanying QCD radiation is $\langle 1-z\rangle M$, where $1-z=1-M^{2} / \hat{s}$ is the average distance from the partonic threshold. When this scale is larger than the jet veto scale $p_{T}^{\text {veto }}$, the effect of the jet veto is expected to be more sizeable. The perturbative instabilities may originate from potentially large logarithmic contributions of the form $\ln (1-z) M / p_{T}^{\text {veto }}$. In our case (with $M=M_{W H}$ being the invariant mass of the $W H$ system) the cuts already select a phase space region in which the radiation recoiling against the $W H$ system is relatively soft, and the additional reduction in the accepted cross section from the jet veto is limited.

As in figure 1 and 2 , in figure 3 (right panel) we plot the $p_{T}$ spectra normalized to the reference NLO prediction, and we study the scale uncertainties. The effect of NLO scale variations is definitely larger than in figure 1 and 2, being of the order of $\mathcal{O}( \pm 15-20 \%)$ in the range considered. We also see that the NNLO uncertainty is smaller than the NLO one, being of $\mathcal{O}( \pm 10 \%)$. We point out that, contrary to what happens without the jet veto (see figure 2), both the NNLO and MC@NLO predictions lie within the NLO uncertainty band. This fact, together with the relatively mild impact of the jet veto on the accepted cross section, gives us confidence that the theoretical prediction is under good control.

In table 1 we report the cross sections and scale uncertainties obtained at the various orders, together with the MC@NLO result. The scale uncertainties are obtained with the procedure discussed above. We note that the MC@NLO uncertainty turns out to be rather small. In the case in which the jet veto is not applied (first row of table 1) this is consistent with what we find at NLO (with LO decay). When a jet veto is applied, the 


\begin{tabular}{|c|c|c|c|c|}
\hline$\sigma(\mathrm{fb})$ & NLO (with LO dec.) & NLO (full) & NNLO (with NLO dec.) & MC@NLO \\
\hline w/o extra jet veto & $1.96_{-1 \%}^{+1 \%}$ & $1.73_{-3 \%}^{+2 \%}$ & $1.56_{-5 \%}^{+5 \%}$ & $1.58_{-1 \%}^{+2 \%}$ \\
\hline w extra jet veto & $1.46_{-8 \%}^{+5 \%}$ & $1.02_{-15 \%}^{+14 \%}$ & $0.87_{-11 \%}^{+11 \%}$ & $1.07_{-1 \%}^{+3 \%}$ \\
\hline
\end{tabular}

Table 1. Cross sections and their scale uncertainties for $p p \rightarrow W H+X \rightarrow l \nu b \bar{b}+X$ at the LHC with $\sqrt{s}=8 \mathrm{TeV}$. The applied cuts are described in the text.
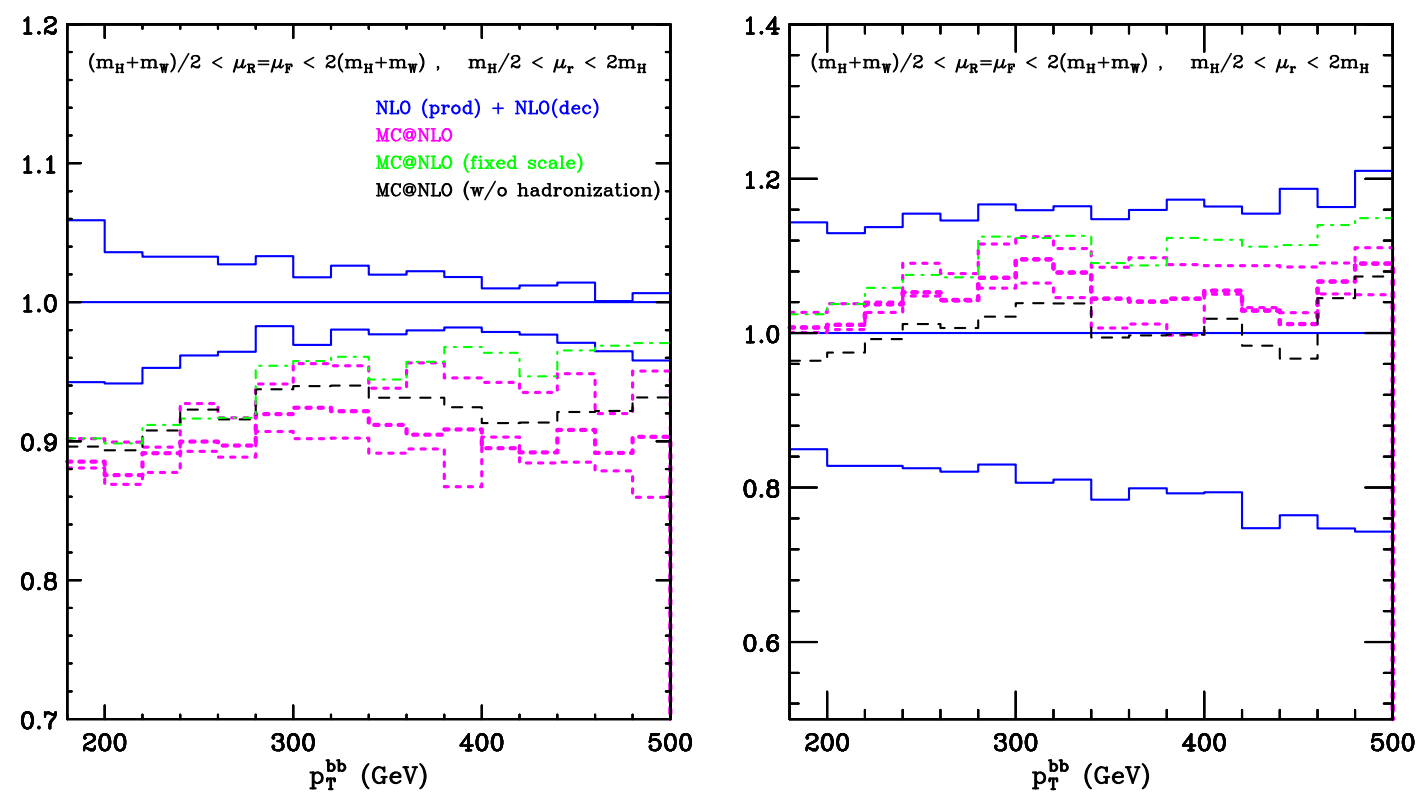

Figure 4. Comparison of NLO with NLO decay (blue solid), MC@NLO with default scale (magenta dots), MC@NLO with fixed scale (green dot-dashes), MC@NLO without hadronization (black dashes). Left panel: without jet veto. Right panel: with jet veto.

MC@NLO uncertainty is still very small, and smaller than the corresponding uncertainty of the NLO result, thus suggesting that it could be underestimated.

In the previous discussion we have compared results for the $p_{T}$ spectrum of the Higgs candidate obtained at different perturbative orders with the result obtained with the MC@NLO event generator, which uses, as default scale, the transverse mass of the WH system, and, besides the effect from the parton shower, includes hadronization. In order to disentangle these different effects in figure 4 we compare the default NLO and MC@NLO results as in figures 2 and 3 , with the MC@NLO result obtained with $\mu_{F}=\mu_{R}=m_{W}+m_{H}$, and with the MC@NLO result without hadronization. The left panel corresponds to figure 2 (no jet veto) and the right panel corresponds to figure 3 (with jet veto). Comparing the MC@NLO result with $\mu_{F}=\mu_{R}=m_{W}+m_{H}$ to the default one, we see that the former is generally consistent with the latter, and tends to lie at the upper edge of the default MC@NLO band at high $p_{T}$. This is consistent with the fact that the fixed scale leads to larger $\alpha_{\mathrm{S}}$ and, as a consequence, larger perturbative corrections at high $p_{T}$. We see that the hadronization effects are relatively small, being at the $1-2 \%$ level in the case in which no jet veto is applied (left panel), and increase to the $5 \%$ level when the jet veto 

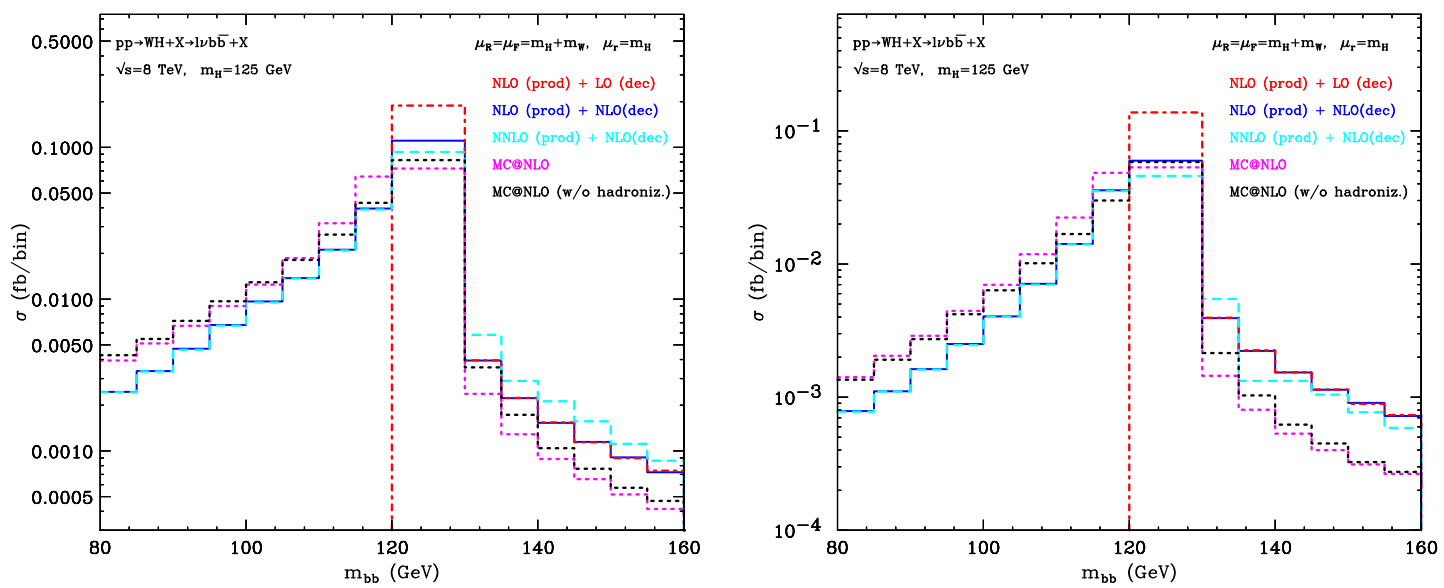

Figure 5. Invariant mass distribution of the pair of $b$-jets computed at NLO with LO decay (red dot-dashes), NLO with NLO decay (blue solid), NNLO with NLO decay (cyan dashes), MC@NLO without hadronization (black dots) and with default MC@NLO (magenta dots). The applied cuts are described in the text. Left panel: without jet veto. Right panel: with jet veto.

is applied (right panel). This is not unexpected: the Higgs $p_{T}$ spectrum is expected to be independent on hadronization if the analysis is sufficiently inclusive. However there is a non trivial relation between the effects of the hadronization and the presence of a jet veto. Indeed, in figure 4 we observe that in the case in which the jet veto is applied (right panel) the contribution of the hadronization has opposite sign with respect to the more inclusive case (left panel). This interplay between hadronization and jet veto would require a dedicated study, which, however, is beyond the scope of the present paper.

We finally consider the invariant mass distribution of the pair of $b$-jets. In figure 5 we plot such distribution at the various perturbative orders and we compare it with the result obtained with MC@NLO. The plot on the left panel corresponds to the case in which the selection cuts discussed above (but no jet veto) are applied; the plot on the right panel is obtained by further applying the light-jet veto.

We start our discussion by noting that when only NLO corrections to the production are considered (dot-dashes histograms in figure 5), the invariant mass distribution is kinematically bounded by $m_{b b} \geq m_{H}$. This is because the parton radiated from the initial state can be clustered in one of the two $b$-jets, thus increasing their total invariant mass. If this is not the case, then we simply have $m_{b b}=m_{H}$. Equivalently, if only NLO corrections to the decay are considered (this case is not shown in figure 5), the invariant mass distribution is bounded by $m_{b b} \leq m_{H}$ because the gluon radiated off the $b \bar{b}$ pair can form a jet on its own, thus decreasing the invariant mass of the dijet system. As already discussed above, in such situations the inclusion of further radiative effects leads to perturbative instabilities [39], which spoil the reliability of the fixed-order expansion around the boundary and would require a resummation of the soft-gluon contributions to all orders. As done in figure 2, we can restore the validity of the fixed-order prediction by choosing a wider bin around the boundary region $m_{b b}=125 \mathrm{GeV}$, which is also the peak region in the invariant mass distribution. 

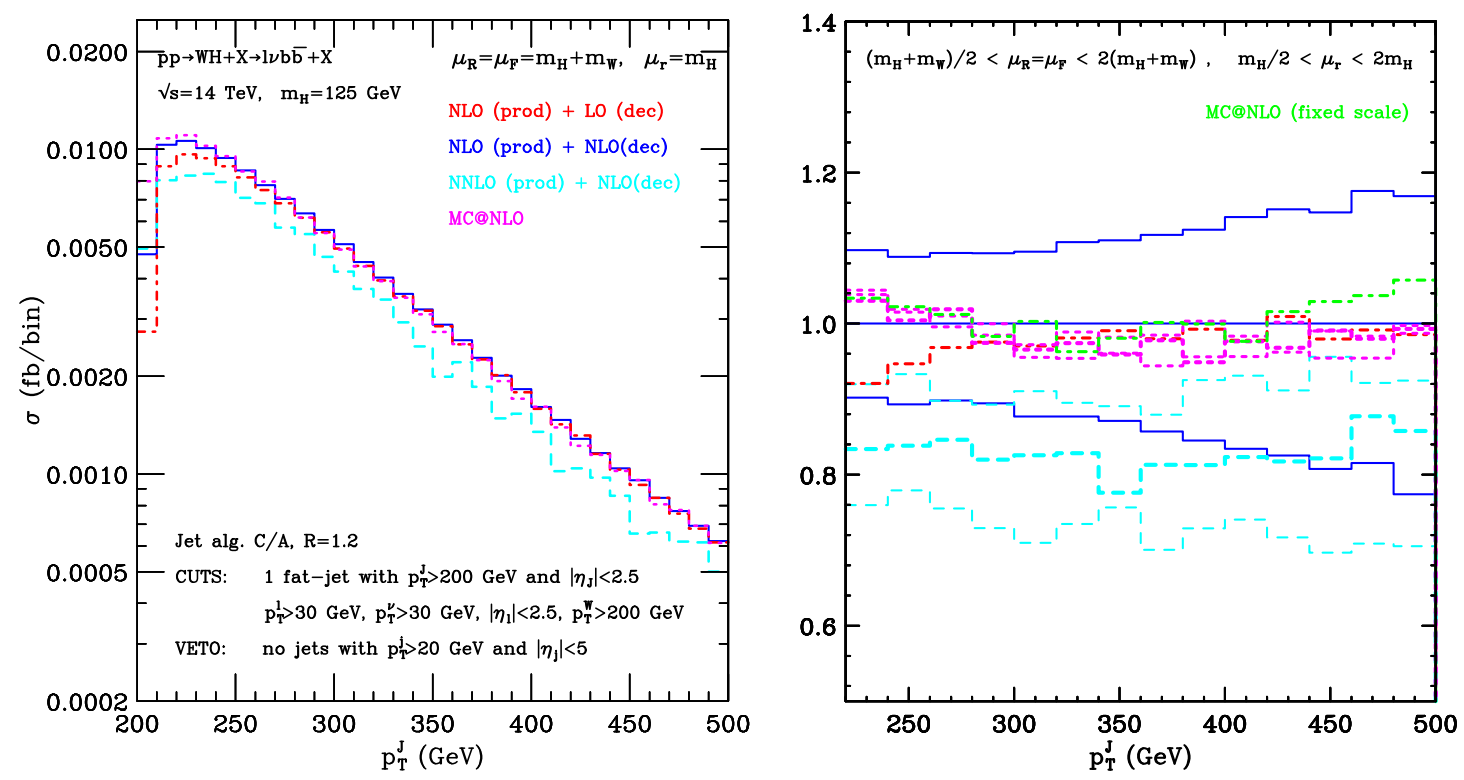

Figure 6. Left panel: transverse-momentum distribution of the fat jet computed at NLO with LO decay (red dot-dashes), NLO with NLO decay (blue solid), NNLO with NLO decay (cyan dashes) and with MC@NLO (magenta dots). Right panel: the same distribution normalized to the full NLO result; the MC@NLO result (green dots) with fixed scale is also shown. The applied cuts are described in the text.

The fact that NLO corrections to production and decay act in opposite regions of the invariant mass spectrum allows us to clearly assess their different impact.

In the high-mass region, NLO corrections to the decay are irrelevant, nevertheless MC@NLO underestimates the cross section. Such effect is due to the parton shower: in the NLO calculation, events in which an initial state parton has been clustered with one of the two $b$-quarks will have $m_{b b}>m_{H}$, but the final state radiation from the parton shower will effectively reduce the dijet invariant mass $m_{b b}$. In this region the NNLO effect is positive, and is partially washed out when the jet veto is applied. In the low-mass region the parton shower is more effective than the fixed order calculations in reducing the invariant mass of the dijet system, and the MC@NLO prediction is higher than the NLO and NNLO result. The effect of hadronization on the MC@NLO result is relatively small: switching off hadronization the difference between the MC@NLO result and the NLO and NNLO results is reduced only partially. In summary, with respect of the MC@NLO prediction, the effect of higher-order QCD corrections is to make the invariant mass distribution harder.

\section{Numerical results at $\sqrt{s}=14 \mathrm{TeV}$}

In this section we consider the case of $W H$ production at the LHC with $\sqrt{s}=14 \mathrm{TeV}$. We follow the selection strategy of ref. [6], that we have already considered in ref. [13]. The Higgs boson is selected at large transverse momenta through its decay into a collimated $b \bar{b}$ pair. 
We require the charged lepton to have $p_{T}^{l}>30 \mathrm{GeV}$ and $\left|\eta_{l}\right|<2.5$, and the missing transverse momentum of the event to fulfil $p_{T}^{\text {miss }}>30 \mathrm{GeV}$. We also require the $W$ boson to have $p_{T}^{W}>200 \mathrm{GeV}$. Jets are reconstructed with the Cambridge/Aachen algorithm [41, 42], with $R=1.2$. One of the jets (fat jet) must have $p_{T}^{J}>200 \mathrm{GeV}^{4}$ and $\left|\eta_{J}\right|<2.5$ and must contain the $b \bar{b}$ pair. In the MC@NLO simulation, the fat jet is required to contain two $B$ hadrons. We also apply a veto on further light jets with $p_{T}^{j}>20 \mathrm{GeV}$ and $\left|\eta_{j}\right|<5$. The corresponding accepted cross sections and uncertainties are reported in table 2 . We see that, compared to the analysis at $\sqrt{s}=8 \mathrm{TeV}$, the effect of the jet veto is more important, and it leads to a reduction of the accepted cross section of about a factor of two for the NLO and MC@NLO predictions, and by $57 \%$ at NNLO. This reduction of the accepted cross section with respect to the case in which the jet veto is not applied is accompanyed by a significant increase in the scale uncertainty in our fixed order results. The reason for this increased sensitivity is twofold: first, the typical invariant mass of the $W H$ system in this case is larger, due to the higher $p_{T}$ required for both the Higgs and the $W$ boson; second, the typical scale of QCD radiation is higher, due to the higher centre-of-mass energy, being the jet veto scale the same used at $\sqrt{s}=8 \mathrm{TeV}$.

Our results for the $p_{T}$ distribution of the Higgs candidate in this boosted scenario are reported in figure 6. Comparing with the results of the previous section we see clear differences. First of all, the effect of NLO corrections for the decay is much smaller, and essentially negligible for $p_{T} \gtrsim 300 \mathrm{GeV}$. This is not unexpected: in this kind of analysis the (boosted) fat jet is essentially inclusive over QCD radiation and the impact of the QCD corrections to the decay is well accounted for by the inclusive QCD corrected $H \rightarrow b \bar{b}$ branching ratio. This observation is important because it confirms the validity of the results presented in ref. [13], where the corrections to the decay were neglected. The NLO scale uncertainty, obtained as in section 3 , is about $\pm 10 \%$ at $p_{T} \gtrsim 200 \mathrm{GeV}$, and it increases to about $\pm 20 \%$ at $p_{T} \sim 500 \mathrm{GeV}$. We also note that the MC@NLO prediction is in good agreement as well with the complete NLO result. In table 2 we see that, as observed in section 3, the MC@NLO prediction has very small uncertainty, much smaller than the scale uncertainties of the other calculations: we thus conclude that, most likely, such uncertainty cannot be considered reliable. The MC@NLO result computed with fixed scale is consistent with the MC@NLO band except in the very high- $p_{T}$ region. The NNLO result is smaller than NLO by about $16 \%$, consistently with what shown in figure 2 of ref. [13], and it is at the border of the band from scale variations. The effect is thus qualitatively similar to what discussed in section 3 but larger in size. The NNLO scale uncertainty band overlaps with the NLO band, and is smaller in size.

In summary, our results on the boosted scenario at $\sqrt{s}=14 \mathrm{TeV}$ show that the shape of the Higgs $p_{T}$ spectrum is rather stable, with uncertainties at the few percent level. The normalization of the accepted cross section has instead larger uncertainties with respect to the analysis at $\sqrt{s}=8 \mathrm{TeV}$. From figure 6 we estimate that these uncertainties are at the $10-15 \%$ level. An alternative way to estimate the perturbative uncertainty could

\footnotetext{
${ }^{4}$ We note that these symmetric cuts on the transverse momenta of the Higgs and the $W$ boson lead to well known perturbative instabilities $[43,44]$ in the fixed order predictions around the cut. Here we simply ignore this problem and focus on the $p_{T}$ distribution of the Higgs candidate sufficiently above $p_{T}=200 \mathrm{GeV}$.
} 


\begin{tabular}{|c|c|c|c|c|}
\hline$\sigma(\mathrm{fb})$ & NLO (with LO dec.) & NLO (full) & NNLO (with NLO dec.) & MC@NLO \\
\hline w/o jet veto & $2.54_{-1 \%}^{+1 \%}$ & $2.63_{-1 \%}^{+1 \%}$ & $2.52_{-2 \%}^{+2 \%}$ & $2.82_{-1 \%}^{+1 \%}$ \\
\hline w jet veto & $1.22_{-14 \%}^{+11 \%}$ & $1.29_{-13 \%}^{+12 \%}$ & $1.07_{-6 \%}^{+8 \%}$ & $1.33_{-1 \%}^{+1 \%}$ \\
\hline
\end{tabular}

Table 2. Cross sections and their scale uncertainties for $p p \rightarrow W H+X \rightarrow l \nu b \bar{b}+X$ at the LHC with $\sqrt{s}=14 \mathrm{TeV}$. The applied cuts are described in the text.

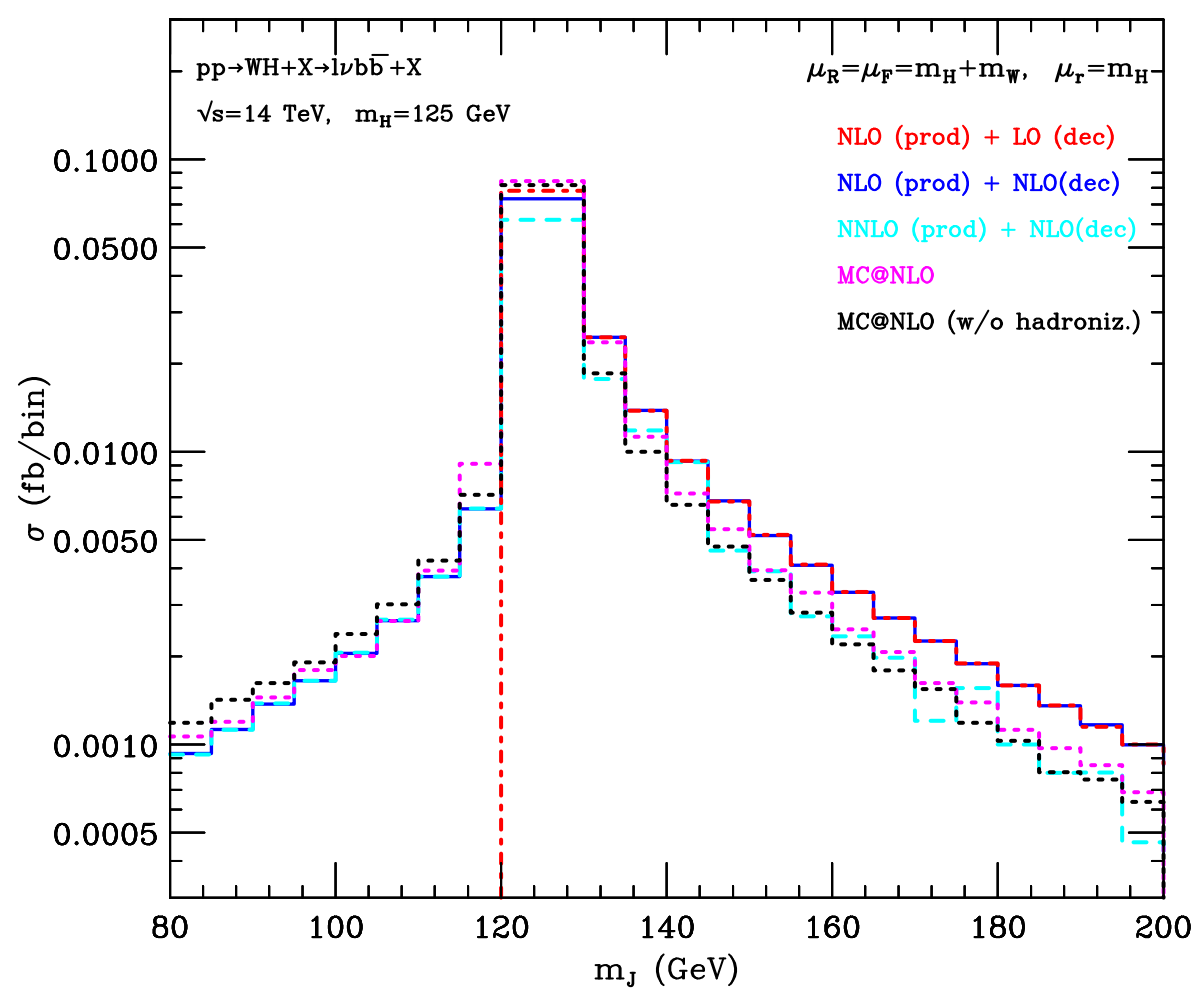

Figure 7. Invariant mass distribution of the fat jet computed at NLO with LO decay (red dotdashes), NLO with NLO decay (blue solid), NNLO with NLO decay (cyan dashes), MC@NLO without hadronization (black dots) and with default MC@NLO (magenta dots).

be to follow the prescription of ref. [45]. A reduction of the uncertainty can be obtained by performing the resummation of the large logarithmic contributions, along the lines of refs. [46-48]. Note, however, that this would be possible at present only by neglecting the radiation from the $b \bar{b}$ pair, whose effect, however, is marginal in the boosted scenario.

We now move to consider the invariant mass distribution of the fat jet. In figure 7 we report our fixed-order predictions for this distribution and compare them to the result from MC@NLO. We immediately see that, contrary to what happens in figure 5, the invariant mass distribution of the fat jet has a more pronounced tail at high mass. This somewhat confirms what we have already observed, that QCD radiative effects on the production process, which naturally populate the high-mass region, are those that are more relevant in the fat-jet analysis. The fixed order and MC@NLO results for $m_{J}<m_{H}$ are essentially identical, whereas at $m_{J}>m_{H}$ the reduction in the cross section due to the parton shower 
is similar in size to the (negative) NNLO effect. From figure 7 we conclude that, contrary to what happens in the analysis at $\sqrt{s}=8 \mathrm{TeV}$ (see section 3), the invariant mass distribution is relatively stable with respect to radiative corrections.

\section{Summary}

In this paper we have studied the effect of QCD radiative corrections on the associated production of the Higgs boson with a $W$ boson in hadronic collisions, followed by the $W \rightarrow l \nu_{l}$ and the $H \rightarrow b \bar{b}$ decays. We performed a QCD calculation that includes the contributions from higher-order radiative corrections up to NNLO for the $W H$ production and up to NLO for the $H \rightarrow b \bar{b}$ decay. By exploiting the narrow-width approximation (see eq. (2.4)) and by appropriately normalizing the $H b b$ coupling, the prediction we obtain is insensitive to higher-order corrections to the $H \rightarrow b \bar{b}$ decay for a completely inclusive observable. Having accounted for the fully exclusive $H \rightarrow b \bar{b}$ decay at the NLO, our calculation should thus provide a reliable approximation to the complete NNLO calculation.

Our computation is implemented in a parton level Monte Carlo program that allows us to apply arbitrary kinematical cuts on the $W$ and $H$ decay products and on the accompanying QCD radiation. A public version of this program will be available in the near future.

We have focused our study on the transverse momentum and the invariant mass distributions of the Higgs candidate, which are the most relevant observables for the experimental analysis at the LHC. We have compared the effects of the QCD radiative corrections at various level of accuracy with the results obtained with the MC@NLO event generator.

We find that NLO corrections to the $H \rightarrow b \bar{b}$ decay can be important to obtain a reliable $p_{T}$ spectrum of the Higgs boson, but that, in the cases of interest, the final state radiation is well accounted for by the Monte Carlo parton shower. The jet veto that is usually applied on additional light jets challenges the stability of the perturbative expansion. Nonetheless, with the selection cuts applied in the $\sqrt{s}=8 \mathrm{TeV}$ analysis, we have shown that the theoretical prediction of $p_{T}$ spectrum of the Higgs candidate appears under good control. The impact of the jet veto is larger in the boosted analysis at $\sqrt{s}=14 \mathrm{TeV}$, and perturbative uncertainties are more sizeable. NNLO corrections to the production process decrease the cross section by an amount which depend on the detail of the applied cuts, but, in all cases we have considered, NNLO corrections have a mild effect on the shape of the Higgs $p_{T}$ spectrum.

The effect of higher-order QCD radiative corrections on the invariant mass distribution of the Higgs candidate is different in the $\sqrt{s}=8$ and $14 \mathrm{TeV}$ analyses. In the $\sqrt{s}=8 \mathrm{TeV}$ analysis the higher-order QCD effects tend to make the invariant mass distribution harder with respect to the $\mathrm{MC} @ \mathrm{NLO}$ prediction. In the fat-jet analysis at $\sqrt{s}=14 \mathrm{TeV}$, the invariant mass of the fat jet is rather stable when higher-order QCD effects are considered.

We finally point out that a possible continuation of the study presented here could be along the lines of refs. $[22,49]$, to perform a full NNLO+PS simulation for this process. 


\section{Acknowledgments}

We would like to thank Stefano Catani, Niklas Mohr and Andrea Rizzi for helpful discussions, Stefano Frixione and Bryan Webber for their help with the MC@NLO event generator, and Andrea Banfi for comments on the manuscript. This research was supported in part by the Research Executive Agency (REA) of the European Union under the Grant Agreement number PITN-GA-2010-264564 (LHCPhenoNet). The work of FT is partially supported by MIUR under project 2010YJ2NYW.

Open Access. This article is distributed under the terms of the Creative Commons Attribution License (CC-BY 4.0), which permits any use, distribution and reproduction in any medium, provided the original author(s) and source are credited.

\section{References}

[1] ATLAS collaboration, Observation of a new particle in the search for the standard model Higgs boson with the ATLAS detector at the LHC, Phys. Lett. B 716 (2012) 1 [arXiv: 1207.7214] [INSPIRE].

[2] CMS collaboration, Observation of a new boson at a mass of $125 \mathrm{GeV}$ with the CMS experiment at the LHC, Phys. Lett. B 716 (2012) 30 [arXiv:1207.7235] [INSPIRE].

[3] P.W. Higgs, Broken symmetries, massless particles and gauge fields, Phys. Lett. 12 (1964) 132 [INSPIRE].

[4] F. Englert and R. Brout, Broken symmetry and the mass of gauge vector mesons, Phys. Rev. Lett. 13 (1964) 321 [INSPIRE].

[5] CDF Collaboration, D0 collaboration, T. Aaltonen et al., Evidence for a particle produced in association with weak bosons and decaying to a bottom-antibottom quark pair in Higgs boson searches at the Tevatron, Phys. Rev. Lett. 109 (2012) 071804 [arXiv: 1207.6436] [INSPIRE].

[6] J.M. Butterworth, A.R. Davison, M. Rubin and G.P. Salam, Jet substructure as a new Higgs search channel at the LHC, Phys. Rev. Lett. 100 (2008) 242001 [arXiv:0802.2470] [INSPIRE].

[7] ATLAS collaboration, Search for the bb decay of the standard model Higgs boson in associated W/ZH production with the ATLAS detector, ATLAS-CONF-2013-079 (2013).

[8] CMS collaboration, Search for the standard model Higgs boson produced in association with a $W$ or a $Z$ boson and decaying to bottom quarks, CMS-HIG-13-012 (2013).

[9] O. Brein, A. Djouadi and R. Harlander, NNLO QCD corrections to the Higgs-strahlung processes at hadron colliders, Phys. Lett. B 579 (2004) 149 [hep-ph/0307206] [INSPIRE].

[10] R. Hamberg, W. van Neerven and T. Matsuura, A complete calculation of the order $\$ \alpha_{s}^{2}$ correction to the Drell-Yan K factor, Nucl. Phys. B 359 (1991) 343 [Erratum ibid. B 644 (2002) 403-404] [INSPIRE].

[11] O. Brein, R. Harlander, M. Wiesemann and T. Zirke, Top-quark mediated effects in hadronic Higgs-strahlung, Eur. Phys. J. C 72 (2012) 1868 [arXiv:1111.0761] [InSPIRE].

[12] S. Dawson, T. Han, W. Lai, A. Leibovich and I. Lewis, Resummation effects in vector-boson and Higgs associated production, Phys. Rev. D 86 (2012) 074007 [arXiv:1207.4207] [INSPIRE]. 
[13] G. Ferrera, M. Grazzini and F. Tramontano, Associated WH production at hadron colliders: a fully exclusive QCD calculation at NNLO, Phys. Rev. Lett. 107 (2011) 152003 [arXiv: 1107.1164] [INSPIRE].

[14] A. Banfi and J. Cancino, Implications of QCD radiative corrections on high-p $p_{T}$ Higgs searches, Phys. Lett. B 718 (2012) 499 [arXiv:1207.0674] [INSPIRE].

[15] M. Ciccolini, S. Dittmaier and M. Krämer, Electroweak radiative corrections to associated WH and ZH production at hadron colliders, Phys. Rev. D 68 (2003) 073003 [hep-ph/0306234] [INSPIRE].

[16] C. Anastasiou, F. Herzog and A. Lazopoulos, The fully differential decay rate of a Higgs boson to bottom-quarks at NNLO in QCD, JHEP 03 (2012) 035 [arXiv:1110.2368] [INSPIRE].

[17] P. Baikov, K. Chetyrkin and J.H. Kuhn, Scalar correlator at $O\left(\alpha_{s}^{4}\right)$, Higgs decay into b-quarks and bounds on the light quark masses, Phys. Rev. Lett. 96 (2006) 012003 [hep-ph/0511063] [INSPIRE].

[18] S. Frixione and B.R. Webber, Matching NLO QCD computations and parton shower simulations, JHEP 06 (2002) 029 [hep-ph/0204244] [INSPIRE].

[19] O. Latunde-Dada, $M C$ and $N L O$ for the hadronic decay of Higgs bosons in associated production with vector bosons, JHEP 05 (2009) 112 [arXiv:0903.4135] [INSPIRE].

[20] S. Frixione, P. Nason and C. Oleari, Matching NLO QCD computations with parton shower simulations: the POWHEG method, JHEP 11 (2007) 070 [arXiv:0709.2092] [INSPIRE].

[21] K. Hamilton, P. Richardson and J. Tully, A positive-weight next-to-leading order monte carlo simulation for Higgs boson production, JHEP 04 (2009) 116 [arXiv:0903.4345] [INSPIRE].

[22] G. Luisoni, P. Nason, C. Oleari and F. Tramontano, $H W^{ \pm} / H Z+0$ and 1 jet at NLO with the POWHEG BOX interfaced to GoSam and their merging within MiNLO, JHEP 10 (2013) 083 [arXiv:1306.2542] [INSPIRE].

[23] K. Hamilton, P. Nason, C. Oleari and G. Zanderighi, Merging $H / W / Z+0$ and 1 jet at $N L O$ with no merging scale: a path to parton shower + NNLO matching, JHEP 05 (2013) 082 [arXiv: 1212.4504] [INSPIRE].

[24] T. Sjöstrand, S. Mrenna and P.Z. Skands, A brief introduction to PYTHIA 8.1, Comput. Phys. Commun. 178 (2008) 852 [arXiv:0710.3820] [InSPIRE].

[25] M. Bahr et al., HERWIG++ physics and manual, Eur. Phys. J. C 58 (2008) 639 [arXiv: 0803.0883] [INSPIRE].

[26] P. Richardson and D. Winn, Investigation of Monte Carlo uncertainties on Higgs boson searches using jet substructure, Eur. Phys. J. C 72 (2012) 2178 [arXiv:1207.0380] [INSPIRE].

[27] LHC Higgs Cross Section Working Group collaboration, S. Dittmaier et al., Handbook of LHC Higgs cross sections: 1. Inclusive observables, arXiv:1101.0593 [INSPIRE].

[28] S. Catani and M. Grazzini, An NNLO subtraction formalism in hadron collisions and its application to Higgs boson production at the LHC, Phys. Rev. Lett. 98 (2007) 222002 [hep-ph/0703012] [INSPIRE].

[29] S. Catani, L. Cieri, G. Ferrera, D. de Florian and M. Grazzini, Vector boson production at hadron colliders: a fully exclusive QCD calculation at NNLO,

Phys. Rev. Lett. 103 (2009) 082001 [arXiv:0903.2120] [INSPIRE]. 
[30] S. Catani, L. Cieri, D. de Florian, G. Ferrera and M. Grazzini, Diphoton production at hadron colliders: a fully-differential QCD calculation at NNLO, Phys. Rev. Lett. 108 (2012) 072001 [arXiv:1110.2375] [INSPIRE].

[31] M. Grazzini, S. Kallweit, D. Rathlev and A. Torre, $Z \gamma$ production at hadron colliders in NNLO QCD, arXiv:1309.7000 [INSPIRE].

[32] S. Catani and M. Seymour, The dipole formalism for the calculation of QCD jet cross-sections at next-to-leading order, Phys. Lett. B 378 (1996) 287 [hep-ph/9602277] [INSPIRE].

[33] S. Catani and M. Seymour, A general algorithm for calculating jet cross-sections in NLO QCD, Nucl. Phys. B 485 (1997) 291 [Erratum ibid. B 510 (1998) 503-504] [hep-ph/9605323] [INSPIRE].

[34] S. Catani, S. Dittmaier, M.H. Seymour and Z. Trócsányi, The dipole formalism for next-to-leading order QCD calculations with massive partons, Nucl. Phys. B 627 (2002) 189 [hep-ph/0201036] [INSPIRE].

[35] E. Braaten and J. Leveille, Higgs boson decay and the running mass, Phys. Rev. D 22 (1980) 715 [INSPIRE].

[36] M. Drees and K.-i. Hikasa, Note on QCD corrections to hadronic Higgs decay, Phys. Lett. B 240 (1990) 455 [Erratum ibid. B 262 (1991) 497] [INSPIRE].

[37] R.D. Ball et al., Parton distributions with LHC data, Nucl. Phys. B 867 (2013) 244 [arXiv: 1207.1303] [INSPIRE].

[38] M. Cacciari, G.P. Salam and G. Soyez, The anti- $k_{t}$ jet clustering algorithm, JHEP 04 (2008) 063 [arXiv:0802.1189] [INSPIRE].

[39] S. Catani and B. Webber, Infrared safe but infinite: soft gluon divergences inside the physical region, JHEP 10 (1997) 005 [hep-ph/9710333] [INSPIRE].

[40] S. Catani, D. de Florian and M. Grazzini, Direct Higgs production and jet veto at the Tevatron and the LHC in NNLO QCD, JHEP 01 (2002) 015 [hep-ph/0111164] [INSPIRE].

[41] Y.L. Dokshitzer, G. Leder, S. Moretti and B. Webber, Better jet clustering algorithms, JHEP 08 (1997) 001 [hep-ph/9707323] [rNSPIRE].

[42] M. Wobisch and T. Wengler, Hadronization corrections to jet cross-sections in deep inelastic scattering, hep-ph/9907280 [INSPIRE].

[43] S. Frixione and G. Ridolfi, Jet photoproduction at HERA, Nucl. Phys. B 507 (1997) 315 [hep-ph/9707345] [INSPIRE].

[44] A. Banfi and M. Dasgupta, Dijet rates with symmetric E(t) cuts, JHEP 01 (2004) 027 [hep-ph/0312108] [INSPIRE].

[45] I.W. Stewart and F.J. Tackmann, Theory uncertainties for Higgs and other searches using jet bins, Phys. Rev. D 85 (2012) 034011 [arXiv:1107.2117] [InSPIRE].

[46] A. Banfi, P.F. Monni, G.P. Salam and G. Zanderighi, Higgs and Z-boson production with a jet veto, Phys. Rev. Lett. 109 (2012) 202001 [arXiv: 1206.4998] [INSPIRE].

[47] T. Becher, M. Neubert and L. Rothen, Factorization and $N^{3} L L_{p}+N N L O$ predictions for the Higgs cross section with a jet veto, JHEP 10 (2013) 125 [arXiv:1307.0025] [INSPIRE].

[48] I.W. Stewart, F.J. Tackmann, J.R. Walsh and S. Zuberi, Jet $p_{T}$ resummation in Higgs production at $N N L L^{\prime}+N N L O$, arXiv:1307.1808.

[49] K. Hamilton, P. Nason, E. Re and G. Zanderighi, NNLOPS simulation of Higgs boson production, CERN-PH-TH-2013-205 (2013). 